\title{
Human Capital Accumulation, Environmental Quality, Taxation and Endogenous Growth *
}

\author{
Manash Ranjan Gupta, \\ Economic Research Unit, \\ Indian Statistical Institute, Kolkata, India. \\ Bidisha Chakraborty ${ }^{\dagger}$ \\ Vijaygarh Jyotish Ray College, \\ Kolkata-700032, India \\ and \\ Economic Research Unit \\ Indian Statistical Institute, Kolkata, India.
}

\begin{abstract}
We consider a Lucas (1988) type model of endogenous growth in which the environmental quality positively affects the rate of human capital accumulation and the environmental quality itself is positively affected by human capital accumulation and is negatively affected by physical capital accumulation. We analyse the effects of taxation on the steady state equilibrium growth rate in this model. We also analyse the transitional dynamic properties of this model.
\end{abstract}

JEL Classification: C62, H23, J24, O15, O41

Keywords: Human Capital, Endogenous growth, Environment, Steady state equilibrium, Saddle Path, Taxes, Abatement Expenditure, Educational Subsidy.

${ }^{*}$ This is a revised version of a paper related to a part of a chapter of the prospective Ph.D. work of the second author which is in progress with the first author in the Indian Statistical Institute, Kolkata. All the remaining errors are of solely ours.

${ }^{\dagger}$ Correspondence to: Economic Research Unit, Indian Statistical Institute, 203, B.T. Road, Kolkata-700108, WestBengal, India. E-mail: bidisha_chakraborty@rediffmail.com; bidisha_isi@yahoo.co.in. Telephone no.:+91-33-24067358, Fax Number:+91-33-25778893 


\section{Introduction}

There exists a substantial theoretical literature focusing on the interaction between the economic growth and the environmental degradation ${ }^{1}$. In these models, the degradation of environmental quality either lowers the utility of the consumer or lowers the productivity of the factors. Most of these models are built in an one sector Ramsey-Solow framework. Environmental degradation is viewed as the social byproduct of the use of modernized machineries in the production sector because the operation of these modenized machines requires the use of pollution enhancing raw materials like oil, coal etc. Some authors like Mohtadi (1996), Bretschger and Smulders (2007), Perez and Ruiz (2007), Hettich (1998) etc. assume a direct relation between the level of environmental pollution and the stock of physical capital when entire physical capital stock is fully utilized ${ }^{2}$. Other authors like Grimaud and Tournemaine (2007), Hettich (1998), Grimaud (1999) etc. assume the level of environmental pollution to be a function of the level of output of the aggregate production sector.

There exists another set of theoretical literature focusing on the role of human capital accumulation on economic growth ${ }^{3}$. The literature starts with the Lucas (1988) model; and this model has been extended and reanalysed by various authors in different directions. The rate of labour augmenting technical progress, i.e., the rate of human capital accumulation is endogenous to the analysis; and the productivity parameter of the human capital accumulation technology is an important determinant of the rate of growth. Some of the works focusing on the interaction between economic growth and environmental pollution are based on the Lucas (1988) framework. In Hettich (1998), environmental pollution negatively affects the welfare of the household; and, in Rosendahl (1996), environmental quality produces a positive effect on the productivity of capital. Ricci (2007) makes a survey of the literature. However, in none of these existing works, except of Gradus and Smulders (1993), environmental quality affects the learning ability of the individuals.

When human capital accumulation is the engine of economic growth, the learning ability of the individual becomes an important determinant of the rate of human capital accumulation. Environmental pollution produces negative effects on the health of the individual; and this lowers the ability to learn. Noise pollution disturbs the academic environment. Margulis (1991) finds significant empirical correlation between lead in air and blood lead levels. Next, he shows that children with higher blood lead levels have a lower cognitive development and requires supplemental education. Kauppi (2006) shows that methyl mercury, whose exposure to human comes

\footnotetext{
${ }^{1}$ See, for example, Mohtadi (1996), Dinda (2005), Gradus and Smulders (1993), Hettich (1998), Rosendahl (1996), Perez and Ruiz (2007), Endress, Roumasset and Zhou (2005), Grimaud(1999), Ricci (2007), Grimaud and Tournemaine (2007) etc.

${ }^{2}$ If capital accumulation means replacement of old machines by more eco-friendly machines, then environmental pollution should vary negatively with capital accumulation.

${ }^{3}$ See for example, Lucas (1988), Rebelo (1991), Bengad (2003), Caballe and Santos (1993), Ortigueira (1998), Faig (1995), Mino(1996), Greiner and Semmler (2002), Alonso-Carrera and FreireSeren (2004), Chamley (1993) etc.
} 
from fish consumption, may lower the learning ability of the children. Air pollution also causes problems related to eye sight and functioning of the brain. Gradus and Smulders (1993) consider this negative effect of environmental pollution in an otherwise identical Lucas (1988) model. However, they do not analyse the effects of various fiscal policies and the transitional dynamic properties of that model.

Human capital accumulation also has a positive effect on the upgradation of the environmental quality. Education makes the people aware of the environmental problems and of the importance of protecting environment; and the educated people can protect the environment in a scientific way. This positive effect of human capital accumulation on the environmental quality is ignored not only by Gradus and Smulders(1993) but also in the other theoretical models like of Mohtadi (1996), Dinda (2005), Hettich (1998), Rosendahl (1996), Ricci (2007) ${ }^{4}$ etc. However, there are empirical supports in favour of this positive relationship. Torras and Boyce (1998) regress environmental pollution on income, on literacy rate, Gini coefficient of income inequality etc; and find that the literacy rate has a significant negative effect on pollution particularly in low income countries. Petrosillo and Zurlini et.al. (2007) find that the attitudes of the tourists, who visit Marine protected area, are highly dependent on their education level. Clarke and Maantay (2006) find that the participation rate of the people in the recycling program counducted in New York city and its neighbourhood is highly dependent on the education level of the participators.

In this paper, we consider a modified version of Lucas (1988) model with two special features. (i)Environmental quality positively affects the marginal return to education; and (ii) Environmental quality varies positively with the stock of human capital and negatively with the stock of physical capital whose full utilization is ensured by the perfect flexibility of factor prices. We analyse the effect of taxation on the steady state equilibrium rate of growth of the economy. The interesting results obtained in this paper are as follows. Firstly, the steady state equilibrium rate of growth, in this model, varies positively with the proportional tax rate imposed on output or on capital income when tax revenue is spent as lumpsum payment. However, this rate of growth is independent of the tax rate imposed on labour income. In Lucas (1988), this rate of growth is independent of the tax rates imposed either on output or on capital income. In Rebelo (1991), the rate of growth varies inversely with the tax rate imposed on output or on capital income. Secondly, there exists unique saddle path converging to the unique steady state equilibrium point. Thirdly, the positive effect of output taxation on the steady state equilibrium rate of growth is strengthened when tax revenue is spent as abatement expenditure. Fourthly, the optimum output tax rate, which is obtained maximizing the balanced rate of growth, varies proportionately with competitive output share of human capital when tax revenue is spent as educational subsidy.

The rest of the paper is organized as follows. Section 2 presents the basic model and contains the analysis of the effect of output taxation on the steady state equilib-

\footnotetext{
${ }^{4}$ Some authors e.g. Grimaud (1999), Goulder and Mathai (2000), Hart (2004) study the issue of environment in R\& D driven growth model where innovations help to improve the environment.
} 
rium rate of growth when tax revenue is distributed as lumpsum payment. Section 3 presents the analysis related to the transitional dynamic properties of the model. Section 4 contains the analysis related to the effects of factor income taxation. In section 5 , we reanalyse the basic model when tax revenue is spent on abatement activity. Section 6 contains the analysis with tax revenue financing the educational subsidy. In section 7 we study the relationship between growth and welfare. Concluding remarks are made in Section 8.

\section{The model}

The model presented in this paper is an extension of Lucas (1988) model. The government imposes a proportional tax on output and the tax revenue is distributed among the individuals as lumpsum payment. The dynamic optimization problem of the representative individual is to maximize

$$
\int_{0}^{\infty} U(C) e^{-\rho t} d t
$$

subject to the production function given by

$$
Y=A(a H)^{\gamma} K^{1-\gamma}
$$

with $A>0$ and $0<\gamma<1$; the dynamic budget constraint given by

$$
\dot{K}=(1-\tau) Y-C+P
$$

with $0 \leq \tau \leq 1$; the human capital accumulation function given by

$$
\dot{H}=m(E)^{\delta}(1-a) H
$$

with $\delta>0$; and the environmental stock accumulation function given by

$$
E=E_{0} K^{-\beta} H^{\beta}
$$

with $E_{0}, \beta>0$. Here $A$ is the technology parameter; $K$ is the stock of physical capital; $H$ is the stock of human capital and $\tau$ is the proportional output tax rate. $E$ is the environmental quality; $P$ is the lumpsum income transfer resulting from the distribution of tax revenue; and $C$ is the level of consumption of the representative household. $Y$ is the Level of output and $a$ is the fraction of labour time allocated to production. $m$ is the productivity parameter in the human capital accumulation function; $\mathrm{u}($.$) is the utility function; \rho$ is the rate of discount and $\gamma$ is the elasticity of output with respect to human capital. Equations (3) and (4) make the present model different from Lucas (1988). Equation (4) with $\beta>0$ implies that environmental quality varies positively with the stock of human capital and negatively with the stock of physical capital. Equation (3) with $\delta>0$ implies that the positive external effect of environmental quality is present in the human capital accumulation function. If $\delta=0$, or $\beta=0$, then we come back to the original Lucas (1988) model. The 
representative individual solves this optimization problem with respect to the control variables $C$ and $a$. $K$ and $H$ are two state variables. However the individual can not internalize the externality.

We assume $U(C)=\ln C$ for the sake of simplicity. We also impose a restriction on the parameters given by

$$
\gamma>\frac{\beta \delta}{1-\beta \delta}
$$

The current value Hamiltonian is given by

$$
Z=\ln C+\lambda_{K}\left[A(1-\tau)(a H)^{\gamma} K^{1-\gamma}-C+P\right]+\lambda_{H}\left[m(E)^{\delta}(1-a) H\right] .
$$

Here $\lambda_{K}$ and $\lambda_{H}$ are the co state variables of $K$ and $H$.

The first order optimality conditions are given by the following.

$$
\frac{\partial Z}{\partial C}=\frac{1}{C}-\lambda_{K}=0,
$$

and

$$
\frac{\partial Z}{\partial a}=\lambda_{K} A(1-\tau) \gamma K^{1-\gamma} a^{\gamma-1} H^{\gamma}-\lambda_{H} m(E)^{\delta} H=0 .
$$

Time behaviour of the co state variables along the optimum growth path should satisfy the following.

$$
\dot{\lambda_{K}}=\rho \lambda_{K}-\lambda_{K} A(1-\tau)(1-\gamma)(a H)^{\gamma} K^{-\gamma},
$$

and

$$
\dot{\lambda_{H}}=\rho \lambda_{H}-\lambda_{K} A(1-\tau) \gamma(a)^{\gamma} H^{\gamma-1} K^{1-\gamma}-\lambda_{H} m(E)^{\delta}(1-a) .
$$

Transversality conditions are given by the followings.

$$
\lim _{t \rightarrow \infty} e^{-\rho t} \lambda_{K}(t) K(t)=\lim _{t \rightarrow \infty} e^{-\rho t} \lambda_{H}(t) H(t)=0 .
$$

The budget of the government is balanced; and hence

$$
P=\tau Y .
$$

Hence, at the aggregate level, equation (2) is modified as follows:

$$
\dot{K}=Y-C .
$$

\subsection{Steady State Equilibrium}

Along the steady state equilibrium growth path, $\frac{\dot{K}}{K}=\frac{\dot{H}}{H}=\frac{\dot{C}}{C}$ and $\frac{\dot{a}}{a}=0$. Using equations (5) and (7), we have

$$
\frac{\dot{C}}{C}=A(1-\tau)(1-\gamma) a^{\gamma}\left(\frac{H}{K}\right)^{\gamma}-\rho .
$$

From the equation (6), we have 


$$
\frac{\lambda_{K}}{\lambda_{H}}=\frac{m(E)^{\delta} H}{A(1-\tau) \gamma K^{1-\gamma} a^{\gamma-1} H^{\gamma}} .
$$

Differentiating both sides of equation (11) with respect to time and then using the steady state equilibrium condition, we have

$$
\left(\frac{H}{K}\right)^{\gamma-\beta \delta}=\frac{m\left(E_{0}\right)^{\delta}}{A(1-\tau) a^{\gamma}(1-\gamma)} .
$$

Along the steady state equilibrium growth path, $\frac{\dot{C}}{C}=\frac{\dot{H}}{H}$. So using equations (3), (4), (10) and (12) we have

$$
a=\left[\frac{\rho^{\gamma-\beta \delta}(A(1-\tau)(1-\gamma))^{\beta \delta}}{m^{\gamma}\left(E_{0}\right)^{\delta \gamma}}\right]^{\frac{1}{(\gamma-\beta \delta-\gamma \beta \delta)}} .
$$

We assume $\gamma>\beta \delta+\gamma \beta \delta$. Thus we find a negative relationship between optimum $a$ and $\tau$ in the steady state growth equilibrium. Note that $a<1$; and this is guaranteed if

$$
\frac{\rho^{\gamma-\beta \delta}(A(1-\tau)(1-\gamma))^{\beta \delta}}{m^{\gamma}\left(E_{0}\right)^{\delta \gamma}}<1 .
$$

Hence, using equations (10), (12) and (13), the balanced growth rate of the economy, denoted by $g$, is obtained as

$$
g=\left[A(1-\tau)(1-\gamma) a^{\gamma}\right]^{-\frac{\beta \delta}{\gamma-\beta \delta}}\left[m\left(E_{0}\right)^{\delta}\right]^{\frac{\gamma}{\gamma-\beta \delta}}-\rho .
$$

Equation (13) shows that $a$ is positively related to the discount rate, $\rho$, production technology parameter, $A$, and the output elasticity coefficient with respect to physical capital, $(1-\gamma)$. This also shows that $a$ is negatively related to the tax rate, $\tau$, productivity parameter of the human capital accumulation function, $m$, and the initial environmental quality, $E_{0}$.

Substituting $A(1-\tau)(1-\gamma)$ from equation (13) in equation (15) we have

$$
g=\rho\left(\frac{1}{a}-1\right) .
$$

So the growth rate, $g$, varies negatively with $a$. As $a$ varies negatively with the tax rate, $\tau$, the growth rate varies positively with the tax rate, $\tau$. So we have the following proposition.

Proposition 1 When human capital accumulation function in the Lucas (1988) model receives the negative external effect from environmental pollution, the steady state equilibrium rate of growth of the economy varies positively with the tax rate on output. 
If either $\delta=0$, or, $\beta=0$ then we go back to the Lucas (1988) model without external effect. In this case

$$
a=\frac{\rho}{m} ; \text { and } g=m-\rho .
$$

Hence the tax rate on output can not affect the growth rate in this case. In the model of Rebelo (1991), the increase in output tax rate reduces the rate of growth of the economy. However, in this present model, the rate of growth varies positively with the output tax rate.

As the tax rate on output is increased, the post tax marginal productivity of physical capital is reduced. This reduces the net rate of return on physical capital; and hence the physical capital is accumulated at a lower rate. As a result, the rate of upgradation of environmental quality is increased. This produces positive external effect on human capital accumulation. So, in the new steady state equilibrium, the rate of growth of human capital as well as the rate of growth of income are increased.

In the model of Rebelo (1991), an increase in the proportional output tax rate causes a decline in the rate of growth. So the optimum tax rate is zero in the Rebelo (1991) model. In Rebelo (1991), physical capital accumulation positively affects the human capital accumulation. So the increase in the output tax rate reduces the rate of growth of human capital and the rate of growth of the economy in the steady state equilibrium. In Mohtadi (1996), an increase in the output tax rate reduces the rate of growth of physical capital. Although there exists negative external effect of physical capital accumulation on the environmental quality in his model, the rate of growth of output is positively related to rate of growth of physical capital accumulation. Hence, in his model, an increase in output tax rate reduces the rate of growth of output. So our result contradicts the results obtained by Rebelo (1991) and Mohtadi (1996). This model points out a case where growth rate is positively related to the tax rate. This is so because physical capital accumulation has no positive effect on the human capital accumulation in this model. If we assume Rebelo (1991) type of human capital accumulation function where physical capital contributes positively to the human capital accumulation and if we also consider the negative effect of physical capital accumulation on environmental quality, then we may not have a monotonic relationship between the growth rate and the tax rate. On the contrary, we may have an interior optimal tax rate which would maximize the balanced growth rate of the economy.

\section{Transitional Dynamics}

We now turn to analyse the transitional dynamic properties of the model around the steady state equilibrium point. We derive the equations of motion which describe the dynamics of the system.

We define two new variables $x$ and $y$ such that $x=\frac{C}{K}$ and $y=\frac{H}{K}$

Using equations (1), (9) and (10) we have

$$
\frac{\dot{x}}{x}=A a^{\gamma} y^{\gamma}[(1-\tau)(1-\gamma)-1]-\rho+x .
$$


Using equations (1), (3), (4) and (9) we have

$$
\frac{\dot{y}}{y}=m E_{0}^{\delta} y^{\beta \delta}(1-a)-A a^{\gamma} y^{\gamma}+x .
$$

Differentiating both sides of the equation (11) with respect to time, t, and then using equations (3), (7), (8) and (9), we have

$$
\frac{\dot{a}}{a}=\frac{\{1-(1-\gamma+\beta \delta)(1-a)\}}{(1-\gamma)} m\left(E_{0}\right)^{\delta} y^{\beta \delta}+A a^{\gamma} y^{\gamma}\left\{\frac{\beta \delta}{1-\gamma}+\tau\right\}-\frac{(1-\gamma+\beta \delta)}{(1-\gamma)} x .
$$

The dynamics of the system is now described by the differential equations (16), (17) and (18). Their solutions describe the time path of the variables $x, y$ and $a$. When either $\beta=0$, or $\delta=0$, and $\tau=0$, these equations of motion become identical to those obtained in Benhabib and Perli (1994).

Along the steady state equilibrium growth path, $\dot{x}=\dot{y}=\dot{a}=0$. Their steady state equilibrium values are denoted by $x^{*}, y^{*}$ and $a^{*}$. From equation (16), we have

$$
x^{*}=\rho-A a^{* \gamma} y^{* \gamma}\{(1-\tau)(1-\gamma)-1\} .
$$

From equations (17) and (18) we have

$$
y^{*}=\left[\frac{A(1-\tau)(1-\gamma) \rho^{\gamma}}{\left(m E_{0}^{\delta}\right)^{1+\gamma}}\right]^{\frac{1}{\beta \delta-\gamma+\gamma \beta \delta}}
$$

and

$$
a^{*}=\left[\frac{\rho^{\gamma-\beta \delta}\{A(1-\tau)(1-\gamma)\}^{\beta \delta}}{\left(m E_{0}^{\delta}\right)^{\gamma}}\right]^{\frac{1}{\gamma-\beta \delta-\gamma \beta \delta}} .
$$

So the steady state equilibrium point is unique. If either $\beta=0$, or, $\delta=0$, then

$$
y^{*}=\left[\frac{A(1-\tau)(1-\gamma) \rho^{\gamma}}{m^{(1+\gamma)}}\right]^{\frac{1}{-\gamma}}
$$

and

$$
a^{*}=\frac{\rho}{m} .
$$

These expressions are similar to those obtained in Benhabib and Perli (1994). We now turn to show that there exists a unique saddle path converging to the unique steady state equilibrium point. Note that it is a system of 3 differential equations. Initial value of the variable, $y$, is historically given; and the values of other two variables $x$ and $a$ can be chosen by the controller. So if the roots are real then, in order to get the unique saddle path converging to the steady state equilibrium point, we need exactly one latent root of the Jacobian matrix corresponding to the system of differential equations to be negative and the other two roots to be positive.

We can show that ${ }^{5}$

Trace of $J=x^{*}+A\left(a^{*} y^{*}\right)^{\gamma} \gamma\left[\tau+\frac{\beta \delta}{(1-\gamma)}-1\right]+m\left(E_{0} y^{\beta}\right)^{\delta}\left[\left(1-a^{*}\right) \beta \delta+\frac{(1-\gamma+\beta \delta)}{(1-\gamma)} a^{*}\right]$;

\footnotetext{
${ }^{5}$ Derivation in detail is shown in the Appendix (A).
} 
and

$$
\text { Det of } J=m\left(E_{0} y^{\beta}\right)^{\delta} x^{*} \frac{\rho}{(1-\gamma)}[\beta \delta(1+\gamma)-\gamma] .
$$

Here $J$ is the $3 \times 3$ Jacobian matrix corresponding to the system of three differential equations.

Since we have assumed $[\beta \delta(1+\gamma)-\gamma]<0$, the Determinant of $J$ is always negative. Note that, if there does not exist any external effect of aggregate human capital on production, then the determinant of the Jacobian matrix of corresponding differential equations in the Lucas (1988) model is always negative. The negative sign of the determinant of $J$ implies that either all the three latent roots are negative or only one root is negative with other two roots being positive. So we have to look at the sign of the trace of $J$. Here the trace of $J$ is positive. So all the roots can not be negative. Hence, only one latent root would be negative and the other two roots would be positive.

Hence, in this case, there is a unique saddle path converging to the unique steady state equilibrium point. So we have the following proposition:

Proposition 2 There exists a unique saddle path converging to the unique steady state equilibrium if $[\beta \delta(1+\gamma)-\gamma]<0$.

Using equations (4), (17) and the definition of $y$, we have

$$
\frac{\dot{E}}{E}=\beta\left[m E_{0}{ }^{\delta} y^{\beta \delta}(1-a)-A a^{\gamma} y^{\gamma}+x\right] .
$$

So it is clear from this differential equation that, once we obtain time behaviour of $y, x$ and $a$ along the unique saddle path, we can easily solve for the intertemporal transitional behaviour of the environmental quality, $E$. Since it is a $3 \times 3$ dynamic system, we can not use the phase diagram to examine the transitional dynamics of environmental quality. However, it is clear that $\frac{\dot{E}}{E}>(<) 0$ for $\frac{\dot{y}}{y}>(<) 0$. So it is the time behaviour of the capital intensity of production, $\frac{H}{K}$, which determines the time behaviour of environmental quality, $E$.

\section{Factor Income Taxation}

Now we consider taxation on factor income at different rates. Suppose that a tax at the rate of $\tau_{K}$ and a tax at the rate of $\tau_{l}$ are imposed on capital income and labour income respectively. If $\tau_{K}=\tau_{l}$, then it is equivalent to taxing output at that rate. The budget constraint of this individual in this case is given by

$$
\dot{K}=\left(1-\tau_{K}\right) r K+\left(1-\tau_{l}\right) w a H-C+P .
$$

Here $r$ and $w$ are rental rate on capital and wage rate respectively. The dynamic optimization problem of the representative individual in this model is to maximize

$$
\int_{0}^{\infty} U(C) e^{-\rho t} d t
$$


with

$$
U(C)=\ln C,
$$

subject to the equations (1), (3), (4) and (19).

The competitive equilibrium conditions of the profit maximizing firm are given by

$$
r=A(1-\gamma)(a H)^{\gamma} K^{-\gamma}
$$

and

$$
w=A \gamma(a H)^{\gamma-1} K^{1-\gamma} .
$$

From the first order optimality conditions ${ }^{6}$, we have

$$
\frac{\dot{C}}{C}=\left(1-\tau_{K}\right) r-\rho
$$

and, in the steady state equilibrium, we have

$$
\left(1-\tau_{K}\right) r=m(E)^{\delta} .
$$

Substituting $r=A(1-\gamma)(a H)^{\gamma} K^{-\gamma}$ in equation (21) we have

$$
\left(\frac{H}{K}\right)^{\gamma-\beta \delta}=\frac{m E_{0}{ }^{\delta}}{\left(1-\tau_{K}\right) A(1-\gamma) a^{\gamma}} .
$$

Equating the rate of growth of consumption to the rate of growth of human capital in the steady state equilibrium, we have

$$
m(E)^{\delta} a=\rho .
$$

Substituting $E$ and $\frac{H}{K}$ from equations (4) and (22) in the equation (23), we have

$$
a=\left[\frac{\rho^{\gamma-\beta \delta}\left(A\left(1-\tau_{K}\right)(1-\gamma)\right)^{\beta \delta}}{m^{\gamma}\left(E_{0}\right)^{\delta \gamma}}\right]^{\frac{1}{(\gamma-\beta \delta-\gamma \beta \delta)}} .
$$

Note that this equation is same as equation (18) with $\tau$ replaced by $\tau_{K}$. $a$ varies inversely with $\tau_{K}$. Also note that $a$ is independent of $\tau_{l}$. Hence $g=\rho\left(\frac{1}{a}-1\right)$ also varies positively with $\tau_{K}$ and is independent of the change in $\tau_{l}$.

Proposition 3 The balanced growth rate of the economy varies positively with the tax rate imposed on capital income and is invariant to the tax rate imposed on labour income.

If the tax rate imposed on physical capital income is increased, then the post tax marginal productivity of capital is reduced. This lowers the rate of growth of consumption. So the rate of growth of physical capital stock is reduced. As a result environmental quality is improved; and this, in turn, exerts positive external effect on human capital accumulation. Hence the rate of growth of human capital is increased. In the steady state growth equilibrium, this causes the rate of growth of output to rise. However, the change in the tax rate on labour income does not affect the marginal productivity of capital; and so it keeps the rate of growth unchanged.

\footnotetext{
${ }^{6}$ The optimality conditions are given in the Appendix B.
} 


\section{Abatement Expenditure}

The abatement expenditure is an important factor determining the quality of environment. In this section, we assume that abatement activity is undertaken by the government. Tax revenue is not distributed as lumpsum payment. It is spent to meet the abatement expenditure denoted by $S$. Hence $S=\tau Y$ and $P=0$. Our modified environmental quality function is given by

$$
E=E_{0} K^{-\beta} H^{\beta-\theta} S^{\theta}
$$

with $\beta>\theta>0$

The budget constraint of the household is given by

$$
\dot{K}=(1-\tau) Y-C .
$$

which is same as equation (2) with $P=0$. The dynamic optimization problem of the representative individual in this model is to maximize

$$
\int_{0}^{\infty} U(C) e^{-\rho t} d t
$$

with

$$
U(C)=\ln C,
$$

and subject to the equations (1), (3), (25) and (26). $S$ is treated as given in the optimization process because it is external to the individual in a competitive economy.

The optimality conditions remain same as obtained in section 2 and hence these are represented again by equations (5), (6), (7) and (8).

Here also we obtain

$$
\frac{\dot{x}}{x}=-A(1-\tau) \gamma a^{\gamma} y^{\gamma}-\rho+x .
$$

Using equations (1), (3), (25) and (26), we have

$$
\frac{\dot{y}}{y}=m E_{0}^{\delta}\left(\tau A a^{\gamma}\right)^{\theta \delta} y^{\delta(\beta+(\gamma-1) \theta)}(1-a)-A(1-\tau) a^{\gamma} y^{\gamma}+x .
$$

Differentiating both sides of the equation (11) with respect to time, $t$, and then using equations (2), (3), (7) and (8), we have

$$
\begin{gathered}
\frac{\dot{a}}{a}=\frac{\{1-\{(1-\gamma)(1-\theta \delta)+\delta \beta\}(1-a)\}}{\{(1-\gamma(1-\theta \delta)\}} m\left(E_{0}\right)^{\delta}\left(\tau A a^{\gamma}\right)^{\theta \delta} y^{\delta(\beta+(\gamma-1) \theta \delta))}+ \\
A(1-\tau) a^{\gamma} y^{\gamma} \frac{\delta[\beta-\theta(1-\gamma)]}{\{1-\gamma(1-\theta \delta)\}}-\frac{\{(1-\gamma)(1-\theta \delta)+\delta \beta\}}{\{1-\gamma(1-\theta \delta)\}} x .
\end{gathered}
$$

In the steady state equilibrium, from equation (27), we have

$$
x^{*}=\rho+A a^{* \gamma} y^{* \gamma}(1-\tau) \gamma .
$$


From equations (28) and (29), we have

$$
y^{*}=\left[\frac{\rho}{(1-\gamma)(1-\tau) A a^{*(\gamma+1)}}\right]^{\frac{1}{\gamma}} ;
$$

and

$$
a^{*}=\left[\frac{\rho^{\gamma-\beta \delta+\theta \delta(1-\gamma)}\{A(1-\tau)(1-\gamma)\}^{(\beta \delta+\theta \delta(\gamma-1))}}{\left(m E_{0}{ }^{\delta} A \tau^{\theta \delta)}\right)^{\gamma}}\right]^{\frac{1}{\gamma+\{\theta-(1+\gamma) \beta)\} \delta}} .
$$

We have already assumed $\beta>\theta$ which implies $\beta>(1-\gamma) \theta$. Hence $a^{*}$ is negatively related to the tax rate and the growth rate, $g$, is positively related to the tax rate. Note that, compared to equation (13), the negative effect of the change in the tax rate on $a^{*}$ becomes stronger in this case. This is so because, firstly, physical capital accumulation has a direct negative effect on environment like that in the previous section. Moreover, abatement expenditure to physical capital ratio also varies negatively with the stock of physical capital because abatement expenditure, being equal to tax revenue, is proportional to income and the average productivity of physical capital is diminishing. So the negative effect of physical capital gets strengthened in this case.

So we have the following proposition.

Proposition 4 Even if the environmental quality varies positively with the abatement expenditure, an increase in the tax rate on output raises the balanced growth rate of the economy.

\section{Educational Subsidy}

In this section, we assume that the tax revenue is spent to finance the educational subsidy only. The modified human capital accumulation function is given by

$$
\dot{H}=m E^{\delta}(1-a) H G^{\phi}
$$

with $\phi>0$. Here

$$
G=\frac{\tau Y}{H}
$$

and $G$ denotes the effectiveness of the educational subsidy that varies positively with the level of subsidy and inversely with the stock of human capital. If $\phi=$ 0 , we come back to the equation (3) of the basic model in section 2 . Since $G$ is proportional to $Y$ and since $Y$ varies positively with capital stock, $K$, the rate of human capital accumulation also receives a positive external effect from physical capital accumulation. The budget constraint of the household is given by equation (26). The representative individual maximizes

$$
\int_{0}^{\infty} \ln C e^{-\rho t} d t
$$

with respect to $C$ and $a$ subject to the equations (1), (4), (26) and (30). $G$ is treated as given in the maximization process. 
We define again $x$ and $y$ such that $x=\frac{C}{K}$ and $y=\frac{H}{K}$. From the definitions of $x$ and $y$ and the optimality conditions ${ }^{7}$ we obtain following equations of motion.

$$
\begin{gathered}
\frac{\dot{x}}{x}=-A(1-\tau) \gamma a^{\gamma} y^{\gamma}-\rho+x, \\
\frac{\dot{y}}{y}=m E_{0}^{\delta}\left(\tau A a^{\gamma}\right)^{\phi} y^{(\beta \delta+(\gamma-1) \phi)}(1-a)-A(1-\tau) a^{\gamma} y^{\gamma}+x,
\end{gathered}
$$

and

$$
\begin{gathered}
\frac{\dot{a}}{a}=\frac{\{1-\{(1-\gamma)(1-\phi)+\delta \beta\}(1-a)\}}{\{\phi \gamma+(1-\gamma)\}} m\left(E_{0}\right)^{\delta}\left(\tau A a^{\gamma}\right)^{\phi} y^{(\beta \delta+(\gamma-1) \phi)}+ \\
A(1-\tau) a^{\gamma} y^{\gamma} \frac{[\beta \delta-\phi(1-\gamma)]}{\{\phi \gamma+(1-\gamma)\}}-\frac{\{(1-\gamma)(1-\phi)+\delta \beta\}}{\{\phi \gamma+(1-\gamma)\}} x .
\end{gathered}
$$

The steady state equilibrium values of the variables are given by

$$
\begin{gathered}
x^{*}=\rho+A a^{* \gamma} y^{* \gamma}(1-\tau) \gamma ; \\
y^{*}=\left[\frac{\rho}{(1-\gamma)(1-\tau) A a^{*(\gamma+1)}}\right]^{\frac{1}{\gamma}} ;
\end{gathered}
$$

and

$$
a^{*}=\left[\frac{\rho^{\gamma-\beta \delta+\phi(1-\gamma)}\{A(1-\tau)(1-\gamma)\}^{(\beta \delta+\phi(\gamma-1))}}{\left(m E_{0}^{\delta}(A \tau)^{\phi}\right)^{\gamma}}\right]^{\frac{1}{\gamma+\phi-(1+\gamma) \beta \delta}} .
$$

Here,

$$
\begin{gathered}
\frac{d a^{*}}{d \tau}=-\left[\frac{\rho^{\gamma-\beta \delta+\phi(1-\gamma)}\{A(1-\gamma)\}^{(\beta \delta+\phi(\gamma-1))}}{\frac{1}{\gamma+\phi-(1+\gamma) \beta \delta}}(1-\tau)^{\frac{\beta \delta(2+\gamma)+(\gamma-2) \phi-\gamma}{\gamma-(1+\gamma) \beta \delta+\phi}}\right. \\
\left(m E_{0}^{\delta} A^{\phi}\right)^{\gamma} \\
\tau^{\frac{(1+\gamma) \beta \delta-\phi-\gamma(1+\phi)}{\gamma-(1+\gamma) \beta \delta+\phi}}\left[\frac{(\beta \delta-\phi) \tau+\phi \gamma}{\gamma-(1+\gamma) \beta \delta+\phi}\right] .
\end{gathered}
$$

If $\beta \delta>(1-\gamma) \phi$, then $a^{*}$ varies negatively with the tax rate. $\beta \delta>(1-\gamma) \phi$ implies that the negative effect of physical capital accumulation generated through environmental degradation outweighs its positive effect generated through subsidization to the human capital accumulation sector. However, if $\beta \delta<(1-\gamma) \phi$, then $a^{*}$ is not monotonically related to the tax rate, $\tau$. Here,

$$
\frac{d a^{*}}{d \tau}>(<) 0 \quad \text { if } \quad \tau>(<) \frac{\phi \gamma}{\phi-\beta \delta} .
$$

So the growth rate maximizing $\left(a^{*} \text { minimizing }\right)^{8}$ tax rate is given by

$$
\hat{\tau}=\frac{\phi \gamma}{\phi-\beta \delta}<1
$$

${ }^{7}$ The optimality conditions are shown in the Appendix C.

${ }^{8}$ Second order condition is also satisfied. See the Appendix (D). 
This optimum tax rate, $\hat{\tau}$, varies positively with $\gamma$ which represents the competitive output share of human capital. This is justified because the tax revenue is spent as educational subsidy. Here $\hat{\tau}=\gamma$ when $\beta=\delta=0$; and $\beta>0$ implies $\hat{\tau}>\gamma$. So in the absence (presence) of the negative effect of environmental degradation on the human capital accumulation, optimum tax rate is equal to (greater than) the competitive output share of human capital.

Proposition 5 If the revenue obtained from the output tax is spent as educational subsidy and if $\beta \delta<(1-\gamma) \phi$, then there exists a unique positive tax rate, $\hat{\tau}$, satisfying $0<\hat{\tau}<1$ and maximizing the steady state equilibrium growth rate. However, this growth rate varies positively with the tax rate when $\beta \delta>(1-\gamma) \phi$.

\section{Growth and Social Welfare}

If the social welfare is a positive function of the balanced growth rate, then there is no conflit between the growth rate maximization and the social welfare maximization. Fortunately, this is true in all the models described in the earlier sections.

Here $W$ stands for the level of social welfare. So

$$
W=\int_{0}^{\infty} \ln C e^{-\rho t} d t=g \int_{0}^{\infty} t e^{-\rho t} d t+\ln C(0) \int_{0}^{\infty} e^{-\rho t} d t=\frac{\ln C(0)}{\rho}+\frac{g}{\rho^{2}}
$$

because $C(t)=C(0) e^{g t}$ along the balanced growth path. In section 2 and in section 4 , where the tax revenue is returned to the individuals as lumpsum income transfer, we have $C(0)=Y(0)-g K(0)$. Hence, we have

$$
\frac{d W}{d g}=\frac{1}{\rho^{2}}-\frac{K(0)}{\rho\{Y(0)-g K(0)\}}=\frac{1}{\rho^{2}}\left[\frac{\frac{C(0)}{K(0)}-\rho}{\frac{C(0)}{K(0)}}\right]
$$

Here, $\frac{d W}{d g}>0$, because

$$
\frac{C(0)}{K(0)}-\rho=\left(m E_{0}^{\delta}\right)^{\frac{-\gamma}{\beta \delta-\gamma+\gamma \beta \delta}} \rho^{\frac{\gamma \beta \delta}{\beta \delta-\gamma+\gamma \beta \delta}}(A(1-\tau)(1-\gamma))^{\frac{\beta \delta}{\beta \delta-\gamma+\gamma \beta \delta}}\left[\frac{1-(1-\tau)(1-\gamma)}{(1-\tau)(1-\gamma)}\right]>0 .
$$

In section 5 and in section 6 , we have $C(0)=(1-\tau) Y(0)-g K(0)$. In this case also, we have

$$
\frac{d W}{d g}=\frac{1}{\rho^{2}}\left[\frac{\frac{C(0)}{K(0)}-\rho}{\frac{C(0)}{K(0)}}\right]
$$

In these two cases, along the steady state growth path, $\frac{C(0)}{K(0)}$ is given by

$$
\frac{C(0)}{K(0)}=\rho+\frac{\gamma \rho}{(1-\gamma) a^{*}}
$$

Hence, in section $5, \frac{C(0)}{K(0)}$ is given by 


$$
\frac{C(0)}{K(0)}-\rho=\frac{\gamma \rho}{(1-\gamma)}\left[\frac{\rho^{\gamma-\beta \delta+\theta \delta(1-\gamma)}\{A(1-\tau)(1-\gamma)\}^{(\beta \delta+\theta \delta(\gamma-1))}}{\left(m E_{0}^{\delta} A \tau^{\theta \delta)}\right)^{\gamma}}\right]^{\frac{-1}{\gamma+\{\theta-(1+\gamma) \beta)\} \delta}}>0
$$

and in section $6, \frac{C(0)}{K(0)}$ is given by

$$
\frac{C(0)}{K(0)}-\rho=\frac{\gamma \rho}{(1-\gamma)}\left[\frac{\rho^{\gamma-\beta \delta+\phi(1-\gamma)}\{A(1-\tau)(1-\gamma)\}^{(\beta \delta+\phi(\gamma-1))}}{\left(m E_{0}^{\delta}(A \tau)^{\phi}\right)^{\gamma}}\right]^{\frac{-1}{\gamma+\phi-(1+\gamma) \beta \delta}}>0
$$

Hence $\frac{d W}{d g}>0$. So the social welfare along the balanced growth path varies positively with the balanced growth rate, $g$. Hence there is no difference between the growth rate maximizing tax rate and the social welfare maximizing tax rate. However, we can not derive the socially optimal tax rate along the transitional growth path ${ }^{9}$.

\section{Conclusion}

We have developed an endogenous growth model where the environmental quality is negatively affected by the accumulation of physical capital and is positively affected by the accumulation of human capital. The rate of human capital accumulation is positively affected by the external effect emanating from environment. The interesting results obtained in this paper are as follows. Firstly, the steady state equilibrium rate of growth, in this model, varies positively with the proportional tax rate imposed on output or on capital income when tax revenue is spent as lumpsum payment. This result holds even if the environmental quality is positively related to the abatement expenditure and the entire tax revenue is spent as abatement expenditure. The optimum tax rate may appear to be finite and positive when the tax revenue is spent as educational subsidy. However, this rate of growth is independent of the tax rate imposed on labour income. In Lucas (1988), this rate of growth is independent of the tax rates imposed either on output or on capital income. In Rebelo (1991), Mohtadi (1995) etc. the rate of growth varies inversely with these tax rates. Garcia Castrillo Sanso (2000), Gomez (2003) find optimal physical capital tax to be zero and optimal labour tax to be positive in the Lucas (1988) model when tax revenue is spent as educational subsidy. But none of these models consider the negative effect of environmental degradation on the human capital accumulation. However, in this model, we have considered the negative effect of environmental degradation on the human capital accumulation and have shown that the steady state equilibrium growth rate would receive a positive effect from taxation either on output or on capital income. Existing literature does not point out such a possibility.

\footnotetext{
${ }^{9}$ The detailed derivations are shown in Appendix E.
} 


\section{References}

Alonso-Carrera J. and M. J. Freire- Seren 2004. Multiple equilibria, fiscal policy and human capital accumulation. Journal of Economic Dynamics and Control. 28 : 841-856

Ben Gad, M., (2003), "Fiscal Policy and Indeterminacy in Models of Endogenous Growth", Journal of Economic Theory, 108, 322-344.

Benhabib,J. and R. Perli, 1994. "Uniqueness and indeterminacy: On the dynamics of endogenous growth". Journal of economic theory, 63,113-142.

Caballe, J. and M. Santos, 1993. On Endogenous Growth with Physical and Human Capital, Journal of Political Economy, 101, 1042-1067.

Clarke, M. and J. Maantay (2006), "Optimizing recycling in all of New York City's neighborhoods: Using GIS to develop the REAP index for improved recycling education, awareness, and participation", Resources, Conservation and Recycling, Volume 46 (2), 128-148.

Chamley, C, 1993. Externalities and dynamics in models of learning or doing, International Economic Review, 34, 583-610.

Dinda, S. (2005), "A Theoretical Basis for Environmental Kuznets Curve" Ecological Economics, 53, 403-413.

Endress, L.H., J. A. Roumasset, T. Zhou (2005). Sustainable growth with environmental spillovers, Journal of Economic Behavior \& Organization, Vol. 58, 527547.

Faig, M. (1995). A Simple Economy with Human Capital: Transitional Dynamics, Technolgy Shocks, and Fiscal Policies. Journal of Macroeconomics, 17, 421-446.

Garcia-Castrillo, P and M. Sanso, 2000. "Human capital and optimal policy in a Lucastype model", Review of Economic Dynamics, 3, 757-770.

Gradus R. and Smulders S.,(1993) "The Tradeoff between Environmental Care and Long Term Growth - Pollution in Three Prototype Growth Models", Journal of Economics, no $1,25-51$.

Gomez, M. A., (2003). "Optimal fiscal policy in the Uzawa-Lucas model with externalities", Economic Theory, 22, 917-925.

Goulder, L.H., Mathai, K., 2000. "Optimal CO2 abatement in the presence of induced 2 technological change". Journal of Environmental Economics and Management 39, 138.

Greiner, A. and W. Semmler. 2002. Externalities of Investment, Education and Economic Growth, Economic Modelling, 19, 709-724.

Grimaud, A (1999). "Pollution Permits and Sustainable Growth in a Schumpeterian Model," Journal of Environmental Economics and Management, Vol 38, 249-266.

Hettich, F. (1998), "Growth Effects of a revenue-neutral Environmental Tax Reform" Journal of Economics, vol67(3), 287-316.

Grimaud, A., 1999. "Pollution permits and sustainable growth in a Schumpeterian Model". Journal of Environmental Economics and Management 38 (3), 249266.

Grimauda, A., F. Tournemaineb, "Why can an environmental policy tax promote growth through the channel of education?" Ecological Economics, Vol 62 (2007) 2736. 
Hart, R., 2004. "Growth, environment and innovation: a model with production vintages and environmentally oriented research". Journal of Environmental Economics and Management 48 (3), 1078 - 1098.

Kauppi, L (2006). "Mercury-background for abating mercury pollution"-Presentation for the International Mercury Conference, Brussels, 26-27 Oct 2006.

Lucas, R. E, (1988), "On the Mechanics of Economic Development," Journal of Monetary Economics, 22, 3-42.

Lucas, R. E, (1990), "Supply-Side Economics: An Analytical Review", Oxford Economic Papers, 1990, 42(2), 293-316.

Margulis, S. (1991), "A Guide to Back of the Envelope Estimates of Environmental Damage Costs: An Application to Mexico." Mimeo, World Bank.

Mino, K, (1996). "Analysis of a Two-Sector Model of Endogenous Growth with Capital Income Taxation", International Economic Review, 37, 227-51.

Mino, Kazuo (2001), "Indeterminacy and Endogenous Growth with Social Constant Returns", Journal of Economic Theory, 97 (1), 203-222.

Mohtadi,H. "Environment, growth and optimal policy design". Journal of Public Economics 63 (1996).119-140.

Ortigueira S, 1998. "Fiscal policy in an endogenous growth model with human capital accumulation - Transitional dynamics with multiple equilibria", Journal of Monetary Economics, 42, 323-355.

Perez, R., J. Ruiz (2007). "Global and local indeterminacy and optimal environmental public policies in an economy with public abatement activities" Economic Modelling, Vol 24, 431452 .

Petrosillo, G. Zurlini, M.E. Corlian, N. Zaccarelli and M. Dadamo (2007), "Tourist perception of recreational environment and management in a marine protected area o Landscape and Urban Planning", Volume 79(1), 29-37.

Rebelo, S, (1991), "Long-run Policy Analysis and Long run Growth," Journal of Political Economy, 99, 500-521.

Ricci, F, (2007), "Channels of transmission of environmental policy to economic growth: A survey of the theory", Ecological Economics, 60 (2007) 688699.

Rosendahl, K.E. "Does improved Environmental Policy Enhance Economic Growth?". Environmental and Resource Economics, 9 (1996). 341-364.

Torras, M and J, Boyce, "Income, inequality, and pollution: a reassessment of the environmental Kuznets Curve", Ecological economics,25, (1998) 147-160.

\section{Appendix A}

Here the Jacobian matrix corresponding to the system of differential equations (16), (17), (18) is given by:

$$
J=\left[\begin{array}{lll}
\frac{\partial \dot{x}}{\partial x} & \frac{\partial \dot{x}}{\partial y} & \frac{\partial \dot{x}}{\partial a} \\
\frac{\partial \dot{y}}{\partial x} & \frac{\partial \dot{y}}{\partial y} & \frac{\partial \dot{y}}{\partial a} \\
\frac{\partial \dot{a}}{\partial x} & \frac{\partial a}{\partial y} & \frac{\partial \dot{a}}{\partial a}
\end{array}\right]
$$


and the elements of the Jacobian matrix evaluated at the steady state equilibrium values of the variables are given as follows.

$$
\begin{gathered}
\frac{\partial \dot{x}}{\partial x}=x^{*} ; \\
\frac{\partial \dot{x}}{\partial y}=A a^{* \gamma} x^{*} \gamma y^{* \gamma-1}\{(1-\tau)(1-\gamma)-1\} \\
\frac{\partial \dot{x}}{\partial a}=A a^{* \gamma-1} x^{*} \gamma y^{* \gamma}\{(1-\tau)(1-\gamma)-1\} ; \\
\frac{\partial \dot{y}}{\partial x}=y^{*} ; \\
\frac{\partial \dot{y}}{\partial y}=m\left(E_{0}\right)^{\delta}(1-a) \beta \delta y^{\beta \delta}-A a^{\gamma} \gamma y^{\gamma} ; \\
\frac{\partial \dot{a}}{\partial y}=\frac{\partial \gamma a^{\gamma-1} y^{\gamma+1}-m\left(E_{0}\right)^{\delta} y^{*(\beta \delta+1)}}{21-(1-\gamma+\beta \delta)(1-a)\}} \\
\frac{\partial \dot{a}}{\partial x}=-\frac{(1-\gamma+\beta \delta) a^{*}}{(1-\gamma)} ;
\end{gathered}
$$

and

$$
\frac{\partial \dot{a}}{\partial a}=\frac{(1-\gamma+\beta \delta)}{(1-\gamma)} m\left(E_{0}\right)^{\delta} y^{\beta \delta} a+\left\{\tau+\frac{\beta \delta}{(1-\gamma)}\right\} A \gamma a^{\gamma} y^{\gamma}
$$

The characteristic equation of the $J$ matrix is given by

$$
\left|J-\lambda I_{3}\right|=0
$$

where $\lambda$ is an eigenvalue of the Jacobian matrix with elements being evaluated at the steady state equilibrium values. The three charateristic roots can be solved from the equation

$$
a_{0} \lambda^{3}+b_{0} \lambda^{2}+a_{1} \lambda+b_{1}=0
$$

where

$$
\begin{gathered}
a_{0}=-1, \\
b_{0}=\text { Trace of } J,
\end{gathered}
$$

$a_{1}=$ sum of the minors of diagonal terms of $J$

and

$$
b_{1}=\text { Determinant of } J
$$


Clearly $a_{0}$ is negative. We can derive that

$$
\begin{aligned}
& b_{0}=\text { Trace of } J=J_{x x}+J_{a a}+J_{y y}=x^{*}+\frac{(1-\gamma+\beta \delta)}{(1-\gamma)} m\left(E_{0}\right)^{\delta} y^{\beta \delta} a+\left\{\tau+\frac{\beta \delta}{(1-\gamma)}\right\} A \gamma a^{\gamma} y^{\gamma} \\
&+ m\left(E_{0}\right)^{\delta}(1-a) \beta \delta y^{\beta \delta}-A a^{\gamma} \gamma y^{\gamma} \\
&=x^{*}+A\left(a^{*} y^{*}\right)^{\gamma} \gamma\left[\tau+\frac{\beta \delta}{(1-\gamma)}-1\right]+m\left(E_{0} y^{\beta}\right)^{\delta}\left[\left(1-a^{*}\right) \beta \delta+\frac{(1-\gamma+\beta \delta)}{(1-\gamma)} a^{*}\right] .
\end{aligned}
$$

Also it can be shown that

$b_{1}=$ Determinant of $J=J_{x x}\left[J_{y y} J_{a a}-J_{y a} J_{a y}\right]-J_{x y}\left[J_{y x} J_{a a}-J_{y a} J_{a x}\right]+J_{x a}\left[J_{y x} J_{a y}-J_{y y} J_{a x}\right]$

$$
=m^{2} E_{0}{ }^{2 \delta} a(1-a) y^{2 \beta \delta} x \beta \delta \frac{(\beta \delta+1-\gamma)}{(1-\gamma)}-A m E_{0}{ }^{\delta} a^{\gamma+1} y^{\gamma+\beta \delta} x(1-\tau) \gamma(1-\gamma)
$$

$+m E_{0}{ }^{\delta} y^{\beta \delta+1} x\left[m E_{0}{ }^{\delta} \beta \delta y^{\beta \delta-1} a \frac{\{1-(\beta \delta+1-\gamma)(1-a)\}}{(1-\gamma)}+A \gamma a^{\gamma+1} y^{\gamma-1}(1-\tau)(\beta \delta-\gamma)\right]$.

From the steady state equilibrium values $x^{*}, y^{*}$ and $a^{*}$ obtained from equations (16), (17), (18) we have the following equations.

$$
A a^{* \gamma+1} y^{* \gamma}=\frac{\rho}{(1-\tau)(1-\gamma)}
$$

and

$$
m E_{0}{ }^{\delta} y^{* \beta \delta}=\frac{A a^{* \gamma} y^{* \gamma}(1-\tau)(1-\gamma)-\rho}{\left(1-a^{*}\right)}
$$

Using the above equations we have

$$
b_{1}=\text { Determinant of } J=\frac{m E_{0}{ }^{*} y^{* \beta \delta} x^{*} \rho}{(1-\gamma)}[\beta \delta(1+\gamma)-\gamma] .
$$

\section{Appendix B}

The current value Hamiltonian function is given by

$$
Z=\ln C+\mu_{K}\left[\left(1-\tau_{K}\right) r K+\left(1-\tau_{l}\right) w a H-C+P\right]+\mu_{H}\left[m(E)^{\delta}(1-a) H\right]
$$

where $\mu_{K}$ and $\mu_{H}$ are the co state variables.

The first order optimality conditions are given by the following.

$$
\frac{\partial Z}{\partial C}=\frac{1}{C}-\mu_{K}=0,
$$

and

$$
\frac{\partial Z}{\partial a}=\mu_{K}\left(1-\tau_{l}\right) w H-\lambda_{H} m(E)^{\delta} H=0 .
$$


Time behaviour of the co state variables along the optimum growth path should satisfy the following.

$$
\mu_{K}=\rho \mu_{K}-\mu_{K}\left(1-\tau_{K}\right) r
$$

and

$$
\dot{\mu_{H}}=\rho \mu_{H}-\mu_{K}\left(1-\tau_{l}\right) w a-\mu_{H} m(E)^{\delta}(1-a) .
$$

Transversality conditions are given by the followings.

$$
\lim _{t \rightarrow \infty} e^{-\rho t} \lambda_{K}(t) K(t)=\lim _{t \rightarrow \infty} e^{-\rho t} \lambda_{H}(t) H(t)=0 .
$$

From equations (B.1) and (B.3) we have

$$
\frac{\dot{C}}{C}=\frac{\left(1-\tau_{K}\right) r-\rho}{\sigma} .
$$

From equation (B.2) we have

$$
\frac{\mu_{K}}{\mu_{H}}=\frac{m E^{\delta}}{\left(1-\tau_{l}\right) w} .
$$

Since $w$ is constant in the steady state, we have

$$
\frac{\dot{\mu_{K}}}{\mu_{K}}=\frac{\dot{\mu_{H}}}{\mu_{H}} \text {. }
$$

From the above equation, we have

$$
\left(1-\tau_{K}\right) r=m(E)^{\delta}
$$

\section{Appendix C}

The dynamic optimization problem of the representative individual in this model is to maximize

$$
\int_{0}^{\infty} U(C) e^{-\rho t} d t
$$

with

$$
U(C)=\ln C
$$

subject to the equations (1), (4), (26) and (30). While maximizing their present discounted value of utility the individual would consider $G$ to be given.

The first order optimality conditions are given by the following:

$$
\frac{\partial Z}{\partial C}=\frac{1}{C}-\lambda_{K}=0,
$$

and

$$
\frac{\partial Z}{\partial a}=\lambda_{K} A(1-\tau) \gamma K^{1-\gamma} a^{\gamma-1} H^{\gamma}-\lambda_{H} m(E)^{\delta} H\left(\frac{\tau Y}{H}\right)^{\phi}=0
$$


Time behaviour of the co-state variables along the optimum growth path should satisfy the following:

$$
\dot{\lambda_{K}}=\rho \lambda_{K}-\lambda_{K} A(1-\tau)(1-\gamma)(a H)^{\gamma} K^{-\gamma}
$$

and

$$
\dot{\lambda_{H}}=\rho \lambda_{H}-\lambda_{K} A(1-\tau) \gamma(a)^{\gamma} H^{\gamma-1} K^{1-\gamma}-\lambda_{H} m(E)^{\delta}(1-a)\left(\frac{\tau Y}{H}\right)^{\phi}
$$

From equations (C.1) and (C.3) we have

$$
\frac{\dot{C}}{C}=(1-\tau)(1-\gamma) A(a H)^{\gamma} K^{-\gamma}-\rho
$$

From equation (C.2) we have

$$
\frac{\lambda_{K}}{\lambda_{H}}=\frac{m E^{\delta}\left(\frac{\tau Y}{H}\right)^{\phi}}{(1-\tau) A \gamma a^{\gamma-1} H^{\gamma-1} K^{1-\gamma}}
$$

Taking logarithm and differentiating both sides of the above mentioned equation with respect to time, $\mathrm{t}$, and then using equations (26), (30), (C.3), (C.4) we have

$$
\begin{gathered}
\frac{\dot{a}}{a}=\frac{\{1-\{(1-\gamma)(1-\phi)+\delta \beta\}(1-a)\}}{\{\phi \gamma+(1-\gamma)\}} m\left(E_{0}\right)^{\delta}\left(\tau A a^{\gamma}\right)^{\phi}\left(\frac{H}{K}\right)^{(\beta \delta+(\gamma-1) \phi)}+ \\
A(1-\tau) a^{\gamma}\left(\frac{H}{K}\right)^{\gamma} \frac{[\beta \delta-\phi(1-\gamma)]}{\{\phi \gamma+(1-\gamma)\}}-\frac{\{(1-\gamma)(1-\phi)+\delta \beta\}}{\{\phi \gamma+(1-\gamma)\}} \frac{C}{K}
\end{gathered}
$$

Using equations (26) and (C.5) we obtain the equation (31). Using equation (C.6) and the definitions of $x$ and $y$ we obtain the equation (33). Using equations (26) and (30) we obtain the equation (32).

\section{Appendix D}

The steady state equilibrium value of $a$ is

$$
a^{*}=\left[\frac{\rho^{\gamma-\beta \delta+\phi(1-\gamma)}\{A(1-\tau)(1-\gamma)\}^{(\beta \delta+\phi(\gamma-1))}}{\left(m E_{0}{ }^{\delta}(A \tau)^{\phi}\right)^{\gamma}}\right]^{\frac{1}{\gamma+\phi-(1+\gamma) \beta \delta}} .
$$

Here,

$$
\begin{gathered}
\frac{d a^{*}}{d \tau}=-\left[\frac{\rho^{\gamma-\beta \delta+\phi(1-\gamma)}\{A(1-\gamma)\}^{(\beta \delta+\phi(\gamma-1))}}{\frac{1}{\gamma+\phi-(1+\gamma) \beta \delta}}(1-\tau)^{\frac{\beta \delta(2+\gamma)+(\gamma-2) \phi-\gamma}{\gamma-(1+\gamma) \beta \delta+\phi}}\right. \\
\left(m E_{0}^{\delta} A^{\phi}\right)^{\gamma} \\
\tau^{\frac{(1+\gamma) \beta \delta-\phi-\gamma(1+\phi)}{\gamma-(1+\gamma) \beta \delta+\phi}}\left[\frac{(\beta \delta-\phi) \tau+\phi \gamma}{\gamma-(1+\gamma) \beta \delta+\phi}\right]
\end{gathered}
$$




$$
\text { Hence, } \frac{d a}{d \tau}=0
$$

when

$$
\tau=\frac{\phi \gamma}{\phi-\beta \delta}=\tau^{*}
$$

$$
\begin{gathered}
\frac{d^{2} a^{*}}{d \tau^{2}} \operatorname{at}\left(\tau=\tau^{*}\right)=\left[\frac{\rho^{\gamma-\beta \delta+\phi(1-\gamma)}\{A(1-\gamma)\}^{(\beta \delta+\phi(\gamma-1))}}{\left(m E_{0}{ }^{\delta} A^{\phi}\right)^{\gamma}}\right]^{\frac{1}{\gamma+\phi-(1+\gamma) \beta \delta}} \frac{\phi \gamma(\phi-\beta \delta-\phi \gamma)}{(\phi-\beta \delta)\{\gamma-(1+\gamma) \beta \delta+\phi\}} \\
\left(1-\tau^{*}\right)^{\frac{\beta \delta(2+\gamma)+(\gamma-2) \phi-\gamma}{\gamma-(1+\gamma) \beta \delta+\phi}-1} \tau^{* \frac{(1+\gamma) \beta \delta-\phi-\gamma(1+\phi)}{\gamma-(1+\gamma) \beta \delta+\phi}}-1
\end{gathered}
$$

This is positive when $\beta \delta<(1-\gamma) \phi$.

\section{Appendix E}

In section 2 and in section 4 , we have

$$
\dot{K}=Y-C .
$$

Hence, along the steady state growth path,

$$
\frac{\dot{K}}{K}=g=\frac{Y(0)}{K(0)}-\frac{C(0)}{K(0)} .
$$

Now, from equation (1) we find that, along the steady state growth path,

$$
\frac{Y(0)}{K(0)}=A a^{* \gamma}\left(\frac{H}{K}\right)^{*} \text {. }
$$

Using equation (10) we have,

$$
g=A(1-\tau)(1-\gamma) a^{* \gamma}\left(\frac{H}{K}\right)^{*^{\gamma}}-\rho
$$

Hence

$$
\frac{C(0)}{K(0)}=\frac{Y(0)}{K(0)}-g=\rho+A a^{* \gamma}\left(\frac{H}{K}\right)^{*^{\gamma}}[1-(1-\tau)(1-\gamma)] .
$$

Substituting the values of $a^{*}$ and $\left(\frac{H}{K}\right)^{*}$ from section 3 we obtain the expression of $\left(\frac{C(0)}{K(0)}-\rho\right)$ in section 7 .

In section 5 and in section 6 , we have

$$
\dot{K}=(1-\tau) Y-C .
$$

Hence,

$$
\frac{C(0)}{K(0)}=(1-\tau) \frac{Y(0)}{K(0)}-g
$$


In these sections also, we find that

$$
g=A(1-\tau)(1-\gamma) a^{* \gamma}\left(\frac{H}{K}\right)^{*^{\gamma}}-\rho .
$$

The expression of $\frac{Y(0)}{K(0)}$ remains same as above. Hence,

$$
\frac{C(0)}{K(0)}-\rho=\frac{\gamma \rho}{(1-\gamma) a^{*}}
$$

